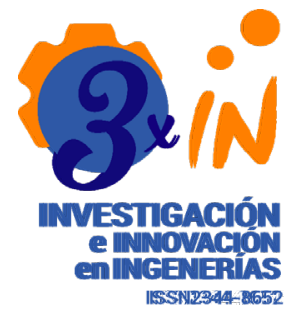

OPEN 2 ACCESS

Recibido: 21/12/2020

Aceptado: 29/03/2021

Publicado: 19/05/2021

Correspondencia de autores: jeisoneduardoep@ufps.edu.co

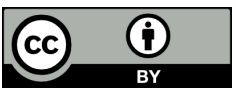

Copyrith 2020

by Investigación e Innovación en Ingenierías

\section{Vehículos aéreos no tripulados como alternativa de solución a los retos de innovación en diferentes campos de aplicación: una revisión de la literatura \\ Unmanned aerial vehicles as an alternative solution to innovation challenges in different fields of application: a literature reviewoperational area of a health center}

\author{
Jeison Eduardo Eslava Pedraza (iD Franyer Adrian Martínez Sarmiento iD \\ Ángelo Joseph Soto Vergel iD \\ Edwin Jose Vera Rozo (iD Dinael Guevara Ibarra iD \\ Universidad Francisco de Paula Santander, Collombia
}

Resumen

Objetivo: RIdentificar los principales campos de aplicación donde los vehículos aéreos no tripulados son una alternativa de solución innovadora, así como las tecnologías implementadas en ellos, articulando información fragmentada. Metodología: Mediante la herramienta Tree of Science se desarrolló una búsqueda inicial en Web of Science y con base en la información obtenida se complementó con el modelo de revisión sistemática tomado como referencia las bases de datos Scopus, ScienceDirec, IEEE y Web of Science aplicando una selección y descarte de información de las diferentes investigaciones relacionadas. Resultados y Conclusiones: Se seleccionaron un total de 81 artículos que fueron analizados y estudiados acorde a las aplicaciones de enfoque donde los vehículos aéreos no tripulados han presentado aportes, soluciones y alternativas a las labores diarias implementadas en áreas como agricultura, ambiente, militar, logística, mapeo y geociencias. Los vehículos aéreos más utilizados como alternativa de solución son los multirrotor que obtuvieron un 58.82 \% de participación, seguido de los ala fija o flexible con un $31.76 \%$. Además, se encontró que el 91.36 \% adaptan cámaras en estas aeronaves para el desarrollo de sus estudios. Finalmente, se observó que el área de la logística, en términos generales, es un tema potencial para estudios futuros.

Palabras clave: Vehículo aéreo no tripulado, UAV, aplicaciones, Tree of Science, revisión sistemática, ala fija, multirrotor.

\section{Abstract}

Objective: identify the main fields of application in which unmanned aerial vehicle are an innovative solution alternative, as well as the technologies implemented in them, articulating fragmented information. Methodology: by the tool Tree of Science was developed a starting research in Web of Science and based on the acquired data was complemented with the review system model took as reference Scopus, ScienceDirect, IEEE and Web of Science databases, applying a selection and discarding of information from the different related investigations. Results and conclusions: It were selected a total of 81 articles that were analyzed and studied according to the approach applications where unmanned aerial vehicles have presented contributions, solutions and alternatives to daily tasks implemented in areas like agriculture, environment, military, logistic, mapping and geoscience. The most widely used air vehicles as an alternative solution are multirotor vehicles, which obtained a $58.82 \%$ share, followed by fixed or flexible wings with $31.76 \%$. In addition, it was found that $91.36 \%$ adapt cameras on these aircraft for the development of their studies. Finally, it was observed that the area of logistics, in general terms, is a potential topic for future studies.

Keywords: Unmanned aerial vehicle, UAV, applications, Tree of Science, systematic review, fixed wing, multirrotor.

Como citar (IEEE): J. Eslava-Pedraza., F. Martínez-Sarmiento., A. Soto-Vergel., E. Vera Rozo., y D. Guevara-Ibarra “Vehículos aéreos no tripulados como alternativa de solución a los retos de innovación en diferentes campos de aplicación: una revisión de la literatura", Investigación e Innovación en Ingenierías, vol. 9, n¹, 149-166, 2021. DOI: https://doi.org/10.17081/invinno.9.1.4017 


\section{Introducción}

Los vehículos aéreos no tripulados (en inglés, Unmanned Aerial Vehicle UAV) han ido presentando un constante crecimiento y desarrollo tecnológico, dando soluciones y facilitando el cumplimiento de actividades en diferentes campos de la ingeniería y afines. Por consiguiente, ha generado la apertura de nuevas líneas de investigación y mejoras en áreas del conocimiento donde están siendo implementados para cumplir labores específicas de monitoreo, vigilancia, seguimiento, búsqueda de personas, prevención de incendios, investigaciones biológicas, manipulación de materiales nocivos y el transporte de pequeños paquetes. Además, el uso de estos dispositivos posibilita la reducción de costos, tiempos de producción y optimización de recursos utilizados en los procesos aplicados a cada campo de trabajo [1], ofreciendo una alternativa innovadora, de labor continua y de alto rendimiento [2].

Si bien el origen de estas tecnologías fue militar, en la última década sus aplicaciones se han extendido a la agricultura, el medio ambiente, geociencia y logística, por mencionar algunas [3, 4, 5]. Estos campos permiten demostrar la versatilidad de estas aeronaves, originando investigación y creación de empresas dedicadas al desarrollo de sistemas y servicios basados en su uso [6]. La mejora continua de estos vehículos está basada actualmente en técnicas de inteligencia artificial (en inglés, Artificial Intelligence - Al) con la finalidad de optimizar las rutas de vuelo mediante generación de nuevos patrones, evasión de obstáculos para prevenir colisiones y reducción del consumo energético para un mayor tiempo de vuelo.

Por lo tanto, este artículo identifica las áreas y campos de aplicación en los cuales los UAV's han presentado mayor aceptación como alternativa de solución innovadora; mostrando indicadores de interés en el análisis bibliográfico. Para ello, realiza el análisis de contenido, evaluación y exposición bibliográfica, enunciando los resultados mediante una clasificación de las aplicaciones identificadas y descripción de las mismas y de las tecnologías encontradas. Finalmente, desarrolla una discusión alrededor de las conclusiones donde se exponen los hallazgos.

\section{Metodología}

La revisión se realiza en idioma inglés y español en una ventana de tiempo delimitada desde 1998 hasta 2019. La Figura 1 detalla las fases metodológicas expuestas para el desarrollo de la revisión: Análisis de contenido, evaluación bibliográfica y exposición bibliográfica.

Figura 1. Metodología para la revisión bibliográfica.

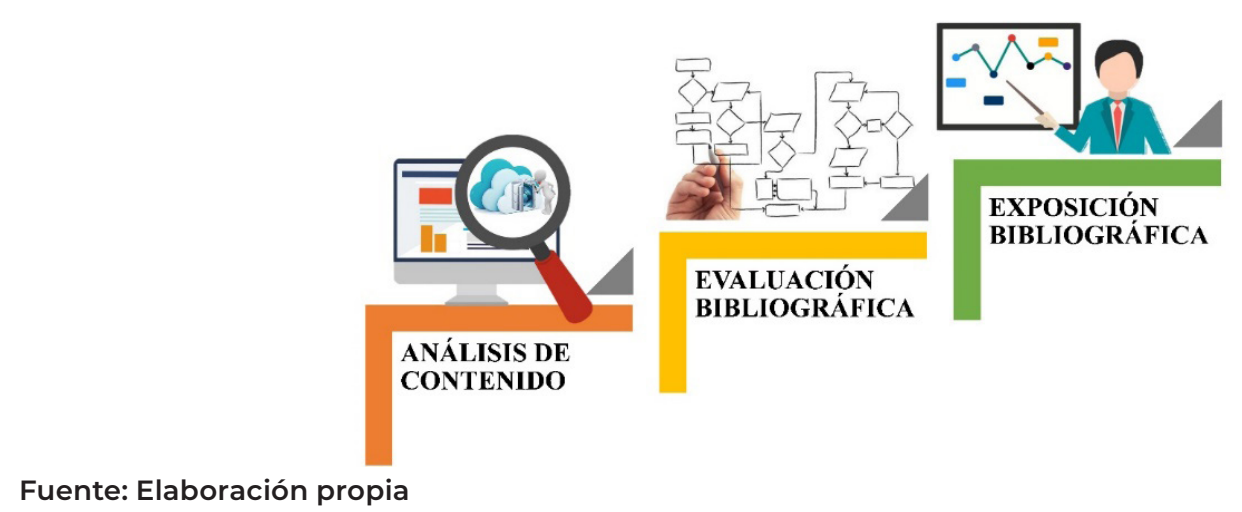


Vehículos aéreos no tripulados como alternativa de solución a los retos de innovación en diferentes campos de aplicación: una revisión de la literatura

\section{Análisis de contenido}

Presenta y selecciona las bases de datos empleadas y las palabras claves para la búsqueda, con la finalidad de exponer de manera circunstancial la cantidad de publicaciones en el área. Se divide en dos fases: Síntesis de la evidencia disponible y búsqueda analítica.

Síntesis de la evidencia disponible. Identifica y selecciona las bases de datos para la revisión, considerando y discriminando por áreas de conocimiento, dando relevancia al área de las ingenierías y afines.

Búsqueda analítica. Define las palabras claves y establece condiciones que delimiten la búsqueda de artículos hasta obtener aquellos que especifiquen los aspectos más relevantes en la investigación. Como resultado se muestra la estadística en años de manera general.

\section{Evaluación bibliográfica}

Selecciona las técnicas de recopilación y distribución de información; para lo cual establece categorías específicas con base al área de investigación y las tecnologías empleadas. Se distribuye en dos fases: Técnicas de recopilación y distribución de la información.

Técnicas de recopilación. Selecciona, define y detalla los modelos de recopilación de información basado en criterios de gestión, clasificación y síntesis a fin de reducir el sesgo, mejorar la calidad y obtener información centrada en el área de investigación.

Distribución de la información. Ejecuta de manera secuencial las técnicas de revisión establecidas, realizando una selección, organización y clasificación de los artículos según los siguientes aspectos:

Selección y descarte. Evalúa los artículos obtenidos mediante el modelo de revisión, aplicando criterios de inclusión y exclusión de artículos, definidos como:

Criterios de inclusión: Cualquier investigación cuyo tema principal sea el desarrollo o empleo de UAV para la realización de labores o actividades específicas. Estas publicaciones deben contar con el Identificador de Objeto Digital (en inglés, Digital Object Identifier DOI).

Criterios de exclusión: Descarta artículos en los que no se presente como énfasis principal el uso de UAV, que tengan poca evidencia investigativa de aplicación real y con resultados similares de otras investigaciones.

Clasificación. Establece los temas fundamentales en los que se fragmenta la revisión bibliográfica según el énfasis de aplicación sustentado en los resultados de los artículos revisados.

\section{Exposición bibliográfica}

Específica cada uno de los temas encontrados en la etapa anterior haciendo énfasis en los desarrollos tecnológicos y presentando sus enfoques investigativos. 


\section{Resultados}

A continuación, se relacionan los resultados obtenidos al aplicar la metodología expuesta anteriormente. Además, se precisan las aplicaciones más relevantes donde estos dispositivos se han empezado a incursionar con estudios e investigaciones en el área, así como también las tecnologías implementadas o acondicionadas a los UAV.

\section{Análisis de contenido}

Se detallan los ítems de síntesis de la evidencia disponible y búsqueda analítica.

Sintesis de la evidencia disponible. Web of Science, Scopus, ScienceDirec y IEEE Xplorer Digital Library son seleccionadas como las bases de datos para la revisión debido a su amplio espectro de publicaciones en investigaciones y estudios, que van desde la electrónica, la instrumentación, las comunicaciones y la informática, hasta áreas afines de la ingeniería por lo que permiten cubrir todos los aspectos necesarios.

Búsqueda analítica. La Figura 2 muestra el modelo de búsqueda general, donde se define detalladamente el método de indagación de la documentación disponible en las bases de datos aplicando como palabra clave "Unmanned Aerial Vehicle", este procedimiento se ejecutó en tres momentos, el primero fue la búsqueda generalizada por tema de investigación, el segundo se realiza por título de artículo y el tercero se delimitó el criterio de búsqueda por título de artículo adicionando la palabra clave "Application".

Figura 2. Modelo de búsqueda general según la base de datos seleccionadas.

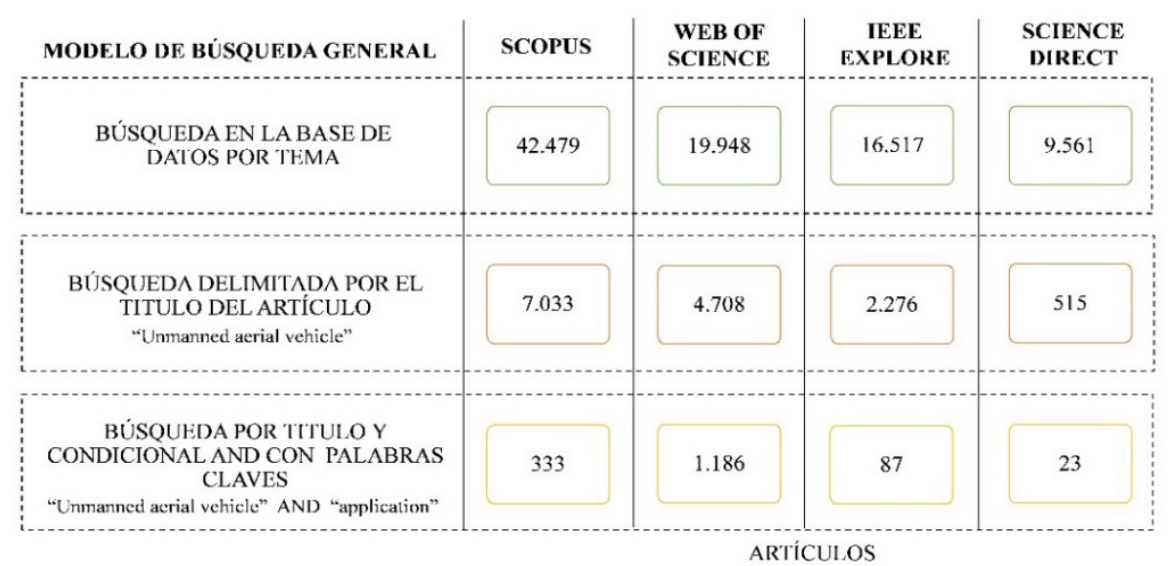

Fuente: Elaboración propia

La Figura 3 presenta el diagrama de tiempos generado con las bases de datos seleccionadas donde se aprecia desde qué año fueron las primeras publicaciones. Además, permite identificar que Scopus y Web of Science son las de mayor recopilación de información acerca del área, por otro lado, se presenta un notable crecimiento investigativo en la última década permitiendo inferir que esta área es aún de gran apogeo en la comunidad científica. 
Figura 3. Diagrama Histórico de la bibliografía seleccionada de la base de datos.

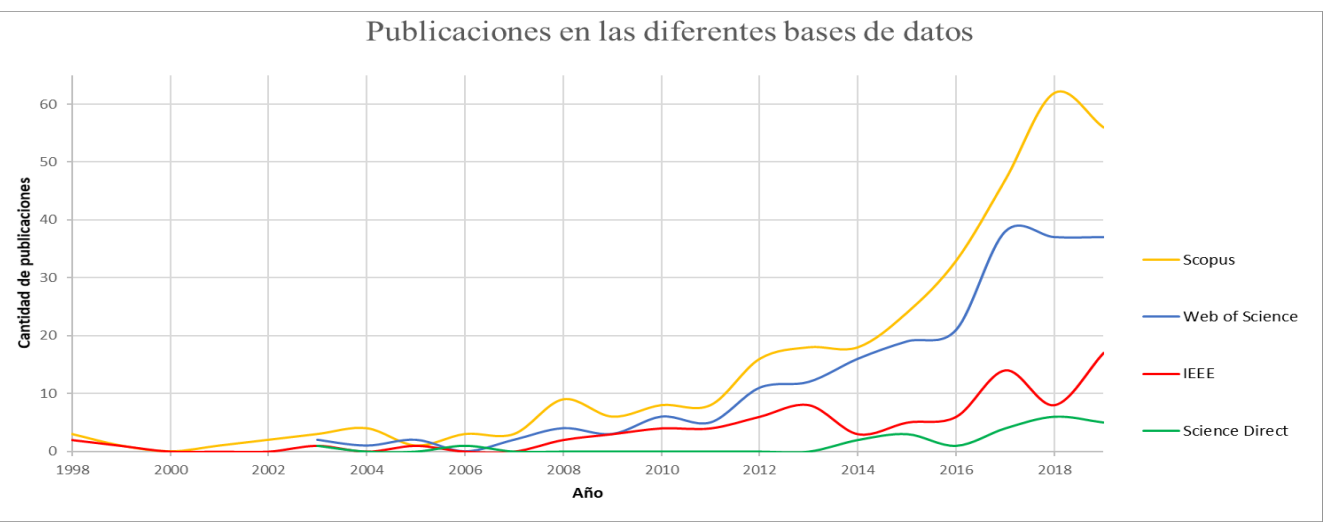

Fuente: Elaboración propia

\section{Evaluación bibliográfica}

A continuación, se presentan aspectos de forma detallada mediante los ítems técnicas de recopilación y técnicas de distribución de información aplicadas para el procedimiento de selección de artículos de mayor relevancia para la investigación.

Técnicas de recopilación. Se selecciona la técnica de análisis de redes mediante una herramienta computacional conocida como Tree of Science para la selección de artículos y la revisión sistemática, dado que son modelos que cumplen con los criterios de reducción de sesgo, presentan una organización clara y facilitan la revisión.

Tree of Science (ToS). Herramienta web que permite seleccionar de manera inteligente artículos científicos aplicando un análisis basado en redes de datos a fin de encontrar contenidos de calidad en temas de investigación [7]. Su método de uso se basa en la plataforma Web of Science, se descargan los metadatos de una búsqueda preliminar para posteriormente ejecutar una búsqueda con los datos obtenidos a fin de organizar un modelo general de documentos organizados en tres bloques Raíz, Tronco y Hojas. En la Raíz se ubican los estudios más clásicos, pero con grandes aportes al conocimiento del área; posteriormente en el Tronco se ubican los artículos que son citados con gran medida, pero citan también a múltiples autores y en las Hojas se ubican los artículos más recientes [8].

Revisión Sistemática. Permite resumir los resultados de los estudios disponibles a fin de recolectar, organizar, evaluar y sintetizar evidencias respecto a un tema de interés [9]. Este Modelo parte de un problema y formula una pregunta muy bien acotada al problema en cuestión; de este modo se permite establecer palabras claves que den solución a la pregunta establecida.[10, 11].

\section{Distribución de la información.}

Tree of Science. A continuación, se enuncian los ítems selección y descarte, y clasificación, detallando los procesos realizados y la cantidad de documentos analizados. 
- Selección y descarte. Mediante las fases expuestas en la Figura 4 se desarrolló la búsqueda y selección de 21 documentos, partiendo desde la búsqueda en Web of Science mediante la herramienta ToS, cuyos artículos fueron filtrados eliminando réplicas de documentación y documentos sin DOI.

Figura 4. Tree of Science, Modelo de búsqueda.

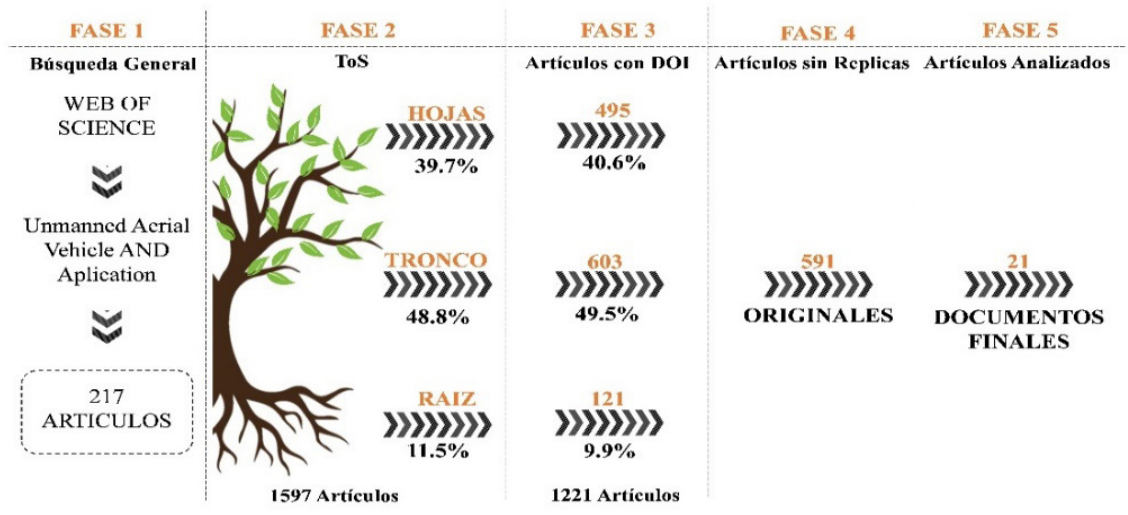

Fuente: Elaboración propia

- Clasificación. Una vez aplicado la selección se clasifican los 21 artículos resultantes por aplicación dividiéndose en agricultura, ambiente, geociencias, milicia y mapeo. La Figura 5 detalla las aplicaciones mencionadas con su respectiva estadística de ocurrencia en los documentos seleccionados. Destacándose que los mayores resultados se encontraron en el sector de la agricultura y el ambiente con esta herramienta de revisión.

Figura 5. Diagrama porcentual según las aplicaciones de los UAV obtenida mediante Tree of Science.

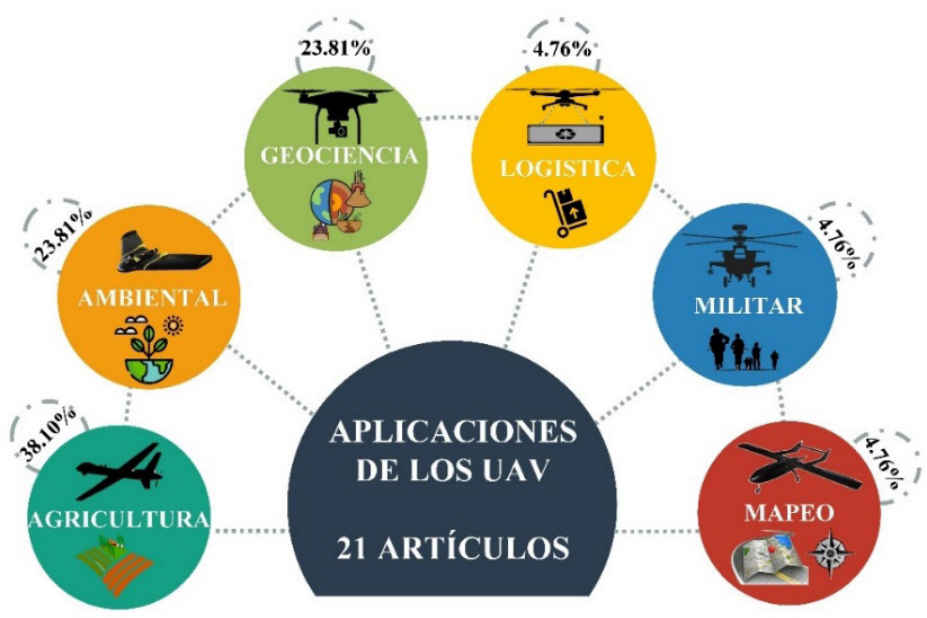

Fuente: Elaboración propia.

\section{Revisión Sistemática}

A continuación, se enuncia los ítems selección y descarte aplicados a la documentación encontrada mediante las técnicas aplicadas y posteriormente el ítem de clasificación estableciendo la relación entre las aplicaciones y la documentación. 
- Selección y descarte. Considerando los resultados obtenidos por el árbol de la ciencia es posible plantear una pregunta problema a fin de refinar la búsqueda del sector de las aplicaciones. ¿Cuáles han sido las principales aplicaciones en las que se usan los UAV? Justificación: La respuesta a esta pregunta permite mostrar con base en las aplicaciones encontradas de los UAV cuales han sido las más usadas y los diferentes avances que han tenido en estas líneas.

Basados en la pregunta generada se realiza una nueva búsqueda con las palabras claves UAV o Unmanned Aerial vehicle y las respectivas aplicaciones considerando componentes claves de cada área. La Figura 6 enuncia las palabras claves utilizadas en el proceso de búsqueda de los artículos en las bases de datos como Web of Science, Scopus, ScienceDirect e IEEE explorer.

Figura 6. Palabras Claves utilizadas en la búsqueda de documentos en las bases de datos.

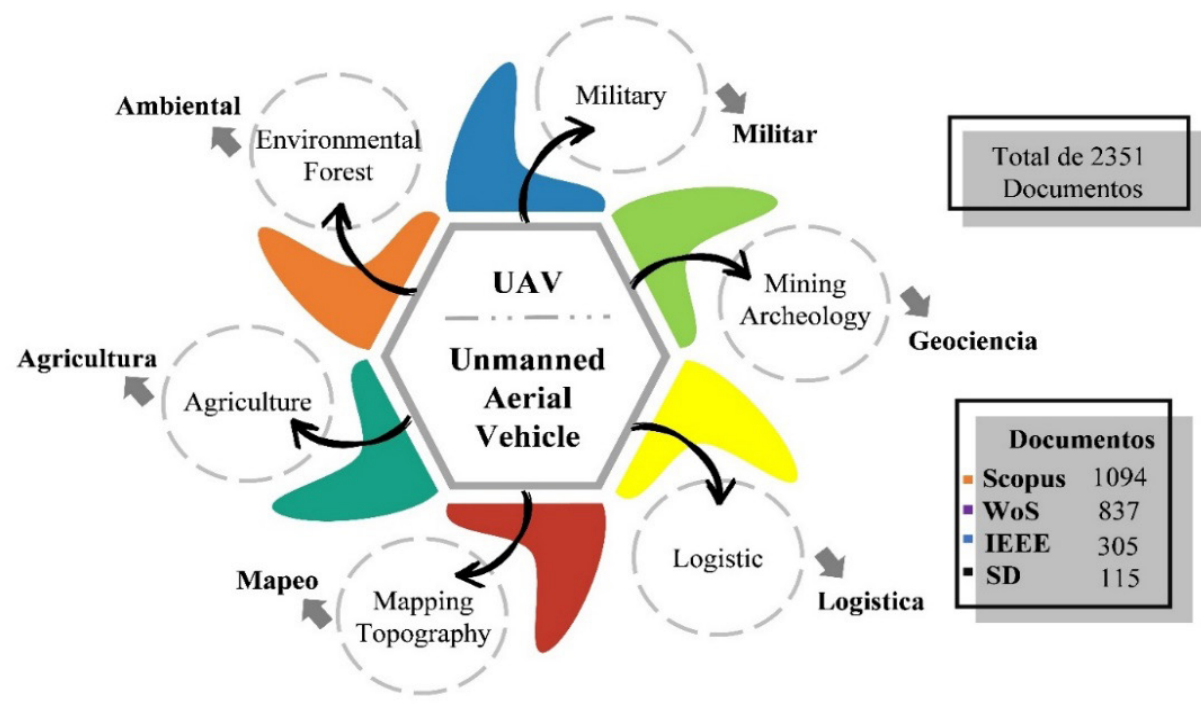

\section{Fuente: Elaboración propia}

Así mismo, la Figura 7 presenta las fases desarrolladas para la selección de los 60 artículos a analizar; presentando las palabras claves seleccionadas y el refinamiento de la búsqueda por base de datos de los 2351 artículos.

Figura 7. Revisión sistemática modelo de búsqueda y selección

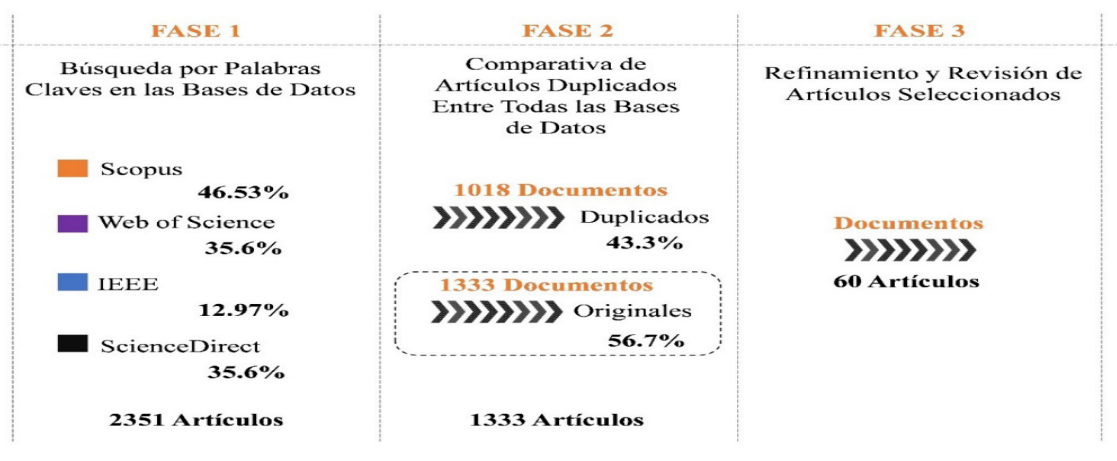

Fuente: Elaboración propia 
- Clasificación. Los 60 artículos seleccionados son clasificados de manera específica en cada una de las secciones respectivas dando lugar a $16.67 \%$ artículos en agricultura, $10 \%$ en militar, $18.33 \%$ en geociencia, 10 \% en logística, $18.33 \%$ en mapeo y $26.67 \%$ en ambiental, además cabe resaltar que el apartado de geociencias se establecieron temas internos respecto a minería y arqueología, en mapeo cartografía y topografía, en ambiental forestal y medio ambiental.

\section{Exposición bibliográfica}

A continuación, se enuncian detalladamente las aplicaciones más relevantes encontradas. Además, la Figura 8 presenta un resumen de las aplicaciones y las principales funciones empleadas por los UAV para el desarrollo de las labores en cada área, así mismo establece la cantidad final de artículos revisados y los porcentajes por cada tópico estudiado.

Figura 8. Estadística general de aplicaciones de los UAV y sus principales funciones.
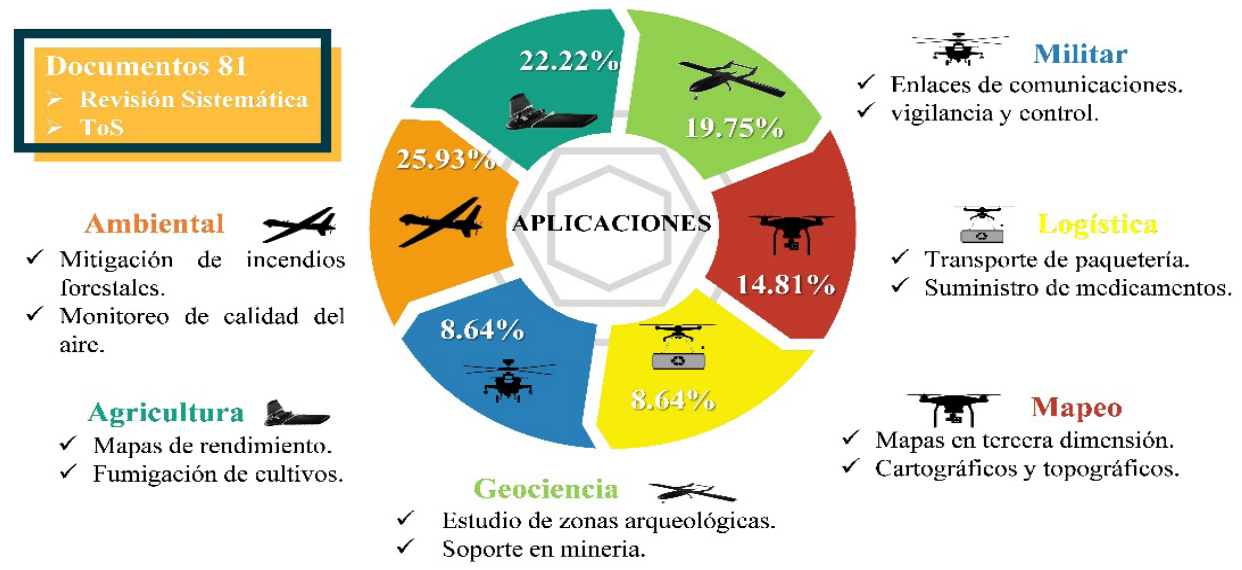

\section{Fuente: Elaboración propia}

Agricultura. En el área de la agricultura los UAV han sido implementados ampliamente, brindando una alternativa a la elaboración, supervisión y control de sistemas agrícolas permitiendo realizar labores manuales desde la identificación del estado actual de un cultivo, hasta el control de crecimiento y cuidado; estos dispositivos incorporan cámaras de alta definición para la adquisición de imágenes [12,13] y aplican métodos como las nubes de puntos y extracción geométrica de formas y técnicas de inteligencia artificial como Redes de Neuronas Artificiales(en inglés, Artificial Neural Networks ANN) y Máquinas de soporte vectorial (del inglés, Support Vector Machine SVM) a fin de generar mapas con características específicas y detalladas de la producción y rendimiento de los cultivos donde se desarrollan gráficas en tercera dimensión del estado actual del cultivo, estimación de la biomasa, predicción del rendimiento y la vigilancia para identificar posibles daños, plagas o maleza en los mismos $[4,14,15]$.

Los UAV no solo han sido empleados como instrumentos de supervisión y monitoreo, sino también han presentado características dentro de sus estructuras como los multirrotor que permiten incorporar herramientas, siendo estas adaptadas en la mayoría de los casos en la parte inferior del vehículo, así como también, sistemas para la detección de tejido vegetal afectado por enfermedades, estructuras para fumigación cultivos y riego en las zanjas de campos y parcelas en diferentes tipos de cultivos, siendo así 
Vehículos aéreos no tripulados como alternativa de solución a los retos de innovación en diferentes campos de aplicación: una revisión de la literatura

dispositivos electrónicos altamente capacitados para facilitar y generar un soporte a las labores realizadas diariamente por los campesinos, debido a que estos se presentan como alternativas para mejorar la productividad de los cultivos [16, 17].

Logística. En el área de la logística los UAV presentan una alternativa para el desarrollo y crecimiento social e industrial ejecutado actividades humanitarias, de mensajería, social y civil. Estos vehículos han realizado actividades y misiones en los últimos años buscando desarrollar modelos a fin de presentar ventajas que permitan a las industrias ser más rentables, empleando tecnologías de bajo costo con alta eficiencia y presentar avances que faciliten la adaptación de nuevas especificaciones en los vehículos utilizados [1].

Los UAV se están implementado para estrategias de transporte de paquetería por empresas multinacionales como Amazon, desarrollando complejos sistemas de entrega incorporando en sus tecnologías algoritmos de optimización de rutas de desplazamiento, establecen en su lógica de control procesos de evasión de obstáculos, la calibración y actualización de las rutas debido a cambios en la ubicación por parte de los usuarios [18, 19]; por otra parte, cuentan con sistemas que son supervisados desde puntos independientes ya sea por el consumidor o por la empresa encargada, utilizando aplicaciones móviles que permiten evaluar el estado del producto, su posición y rumbo en tiempo real, donde el usuario puede estar informado del estado de sus productos [20].

Sin embargo, el uso particular de los UAV busca ampliar el rango de trabajo de estos dispositivos para la evaluación y monitoreo de los flujos de tráfico peatonal en zonas urbanas estableciendo puntos estratégicos como plazas de mercados, centros comerciales, parques, entre otros; con el fin de identificar patrones, gestionar la demanda y la oferta de los peatones en estas ubicaciones que son objeto de estudio [21]. Por otra parte, han sido objeto de investigaciones en el área de logística humanitaria brindando ayuda a personas durante y después de desastres naturales, siendo alternativas que favorecen a los organismos encargados en prevención y atención para distribuir suministros, además son empleados para la búsqueda y rescate de personas aplicando algoritmos de metaheurísticas para la reducción del desplazamiento entre los puntos de control y los de apoyo, y entre las estaciones y zonas de difícil acceso que se puedan observar durante una catástrofe natural [22].

Militar. El origen de los UAV parte en esta área de acción, teniendo pruebas iniciales en misiones tácticas y de reconocimiento, actualmente son empleados en labores de control de inmigrantes, contrabando y comunicaciones [23]. Estos Vehículos han presentado avances con el desarrollado de sistemas integrados basados en dispositivos electrónicos como sensores, cámaras, radares, Sistemas de Posicionamiento Global (en inglés, Global Positioning System GPS) y el Sistema Global para las Comunicaciones Móviles (del inglés, Global System for Mobile communications GSM) dando calidad a las operaciones realizadas en la milicia, algunas misiones se enfocan en las comunicaciones donde el UAV toma mayor relevancia debido a que genera una conexión y estabilidad de redes inalámbricas entre bases y brigadas, retransmisiones de radio con enlaces de datos tácticos punto a punto de nivel superior (en inglés, Top-Level Domain TLD), trabajando en bandas de frecuencias altas debido a su velocidad de procesamiento de datos, estas labores se llevan a cabo gracias a las características de vuelo y a su clasificación, en UAV tácticos, de resistencia, de altitud media y alta. Sin embargo, estos vehículos aéreos se han implementado para identificar fallas o daños producidos por automóviles y camiones de carga en las líneas de comunicaciones militares, ejecutando monitoreo en tiempo real con algoritmos de aprendizaje automático para la detección de peligros ocultos en zonas aledañas a las líneas de comunicación [24, 25]. 
No obstante, estos vehículos siguen evolucionando e incorporando nuevas tecnologías como los modelos de control y desplazamiento multimodales, por voz y controles por aplicativos táctiles reduciendo la cargar de control de los operarios y facilitando el desarrollo de múltiples tareas, delegando instrucciones a los UAV, agilizando procesos y ejecutando misiones simultáneas de forma rápida y práctica [26, 27]. Así mismo, los UAV están siendo implementados en zonas urbanas densamente pobladas y de alta concurrencia vehicular y peatonal, sirviendo como apoyo a las fuerzas públicas para establecer el orden durante protestas y disturbios civiles, puesto que estos son manipulados o monitoreados por operarios que identificaran entre un civil y un objetivo militar durante estas labores de orden público en las ciudades [28].

Ambiental. El desarrollo de UAV se han acoplado en el área ambiental para mitigar las problemáticas existentes en este sector, empleados como dispositivos para el sensado, supervisión, prevención y cuidado de ecosistemas, bosques y animales. Partiendo desde la evaluación del estado actual del medio ambiente en donde sobrevuelan las áreas y adquieren información detallada mediante sensores térmicos, laser, biosensores y cámaras multiespectrales de las condiciones ambientales, climatológicas, estrés hídrico, calidad del agua como el PH de ríos, lagos y represas [29,30], además de la evaluación de la biomasa, empleando técnicas de procesamiento a las diferentes tomas de imágenes y filtros adicionales para mejorar y aclarar los detalles de las tomas en los bosques tropicales [31, 32]. Los UAV también han sido aplicados con frecuencia para evaluar la vida silvestre e identificar la abundancia animal empleando técnicas de monitoreo con sensores infrarrojos, estos drones permiten realizar operaciones seguras a bajas altitudes de vuelo y en horarios nocturnos cuando la visibilidad es nula [33, 34].

Por otro lado, el uso de los UAV ha orientado investigaciones al sector forestal empleados en la supervisión del estado actual de los bosques [35], predicción y prevención de posibles plagas en la vegetación y su ecosistema. Además, han servido de ayuda para el cuidado de zonas explotadas y tratadas para la extracción del petróleo, permitiendo una vigilancia en sus procesos de reforestación [36, 37]. Los UAV han facilitado el desarrollando de dispositivos para la supervisión y control de incendios forestales, control de desastres naturales y peligros sobre acantilados rocosos, empleando técnicas de adquisición y procesamiento de imágenes multiespectrales, detección de monóxido y dióxido de carbono aplicando aprendizaje autónomo supervisado e implementación de Big data para la detección de la neblina o incendios en una etapa temprana $[38,39]$.

Geociencias. En la geociencia los UAV se presentan como una herramienta práctica y alternativa para la evaluación de zonas con recursos minerales, estructuras rocosas y restos de edificaciones antiguas como túmulos funerarios, arquitecturas antiguas, asentamientos, entre otros, estas aeronaves gracias a sus diseños incorporan en su estructura sistemas con sensores de posicionamiento para la ubicación y referenciación, sensores infrarrojos térmicos, LIDAR y cámaras RGB digitales con las cuales realizan escaneos en zonas específicas y potenciales para la obtención de restos arqueológicos. En consecuencia, los UAV se implementan para ejecutar estas actividades con frecuencia obteniendo datos e información que son procesados aplicando técnicas para el reconocimiento de formas en la tierra con algoritmos de inteligencia artificial (IA) y de estructura de movimiento (en inglés, Structure from Motion SFM) a fin de obtener gráficos 3D geofísicos y mapas binarios del área estudiada [40], estas características han dado a conocer el desarrollando de dispositivos de bajo costo, con precisión y calidad similar a las técnicas tradicionales empleadas en estas labores [41].

Por otra parte, los UAV han incursionado en labores de inspección a través de modelos inteligentes de desplazamiento en tiempo real, realizando vuelos sobre zonas mineras y sus alrededores, integrando planificación de movimientos basado en algoritmos de trayectorias rápidas para la exploración de árboles aleatorios (en inglés, Rapidly Exploring Random Trees RRT) a fin de monitorear la rehabilitación de las áreas 
en las que se llevan a cabo los procesos de extracción minera. Además, tienen capacidad para estimar volúmenes de extensiones de tierra, estabilidad y hundimiento en donde se llevan a cabo procesos de minería a cielo abierto y subterráneos [42, 43]. Obtienen mediciones de concentraciones de químicos como el CO2, el Fe y el índice de vegetación de diferencia normalizada (en inglés, Normalized Difference Vegetation Index, NDVI) que son emitidos y encontrados en el ambiente, estos se obtienen mediante métodos de regresión de mínimos cuadrados parciales y redes neuronales a fin de generar mapas de concentraciones de estos químicos [44]. Finalmente, estos vehículos han cumplido misiones estratégicas para la recuperación, apoyo, búsqueda y rescate en derrumbes o fallas que se puedan presentar durante las labores realizadas en minas, debido a que son actividades complicadas y riesgosas, en consecuencia estos vehículos han permitido que se realicen pruebas de funcionamiento para realizar operaciones y descender a través de túneles mineros en busca del personal estableciendo sistemas de telecomunicaciones interconectados de forma constante con los dispositivos [45].

Mapeo. Los UAV han sido utilizados para la generación de mapas haciendo énfasis en aplicaciones Topográficas y de Cartografía donde los ingenieros y arquitectos ven la utilidad de estos dispositivos para la evaluación de zonas para posibles edificaciones [46], generando mapas de alta resolución y de precisión significativa mediante la creación de diagramas de planificación urbana, topografía y catastral con modelos en tercera dimensión, mediante sensores y cámaras multiespectrales [47]. No solo el UAV ha sido empleado para mapas de tierra, sino también para el estudio de cuencas hidrográficas evaluando la calidad del agua mediante recorridos durante el sector adquiriendo datos necesarios para la investigación [48, 49].

Por otra parte, este tipo de vehículos han ejecutado labores para la evaluación de las pendientes en estructuras y zonas costeras mediante cámaras digitales y sensores de navegación inercial, barómetro y magnetómetro; estableciendo rutas de vuelo óptimas que facilitan la adquisición de imágenes de alto grado de superposición aplicando nubes de puntos de control de tierra con el objetivo de proporcionar información topográfica 3D completa y precisa de forma automática y de alta eficiencia [50]. En otros casos han aportado estrategias para evaluar la topografía resultante en deslizamientos o desastres naturales implementando estos dispositivos como una herramienta ágil y rápida en la gestión y determinación del estado estructural de un terreno mediante escaneos en tercera dimensión que a comparación de las técnicas terrestres mediante datos LIDAR, presentan nuevas formas de análisis fiables y precisas con óptimos resultados [51].

Tecnologías en los UAV. La Figura 9, presenta los sensores y dispositivos electrónicos incorporados con mayor frecuencia en los UAV a fin de mejorar características circunstanciales para el desplazamiento y el sensado. 
Figura 9. Dispositivos electrónicos implementados en los UAV

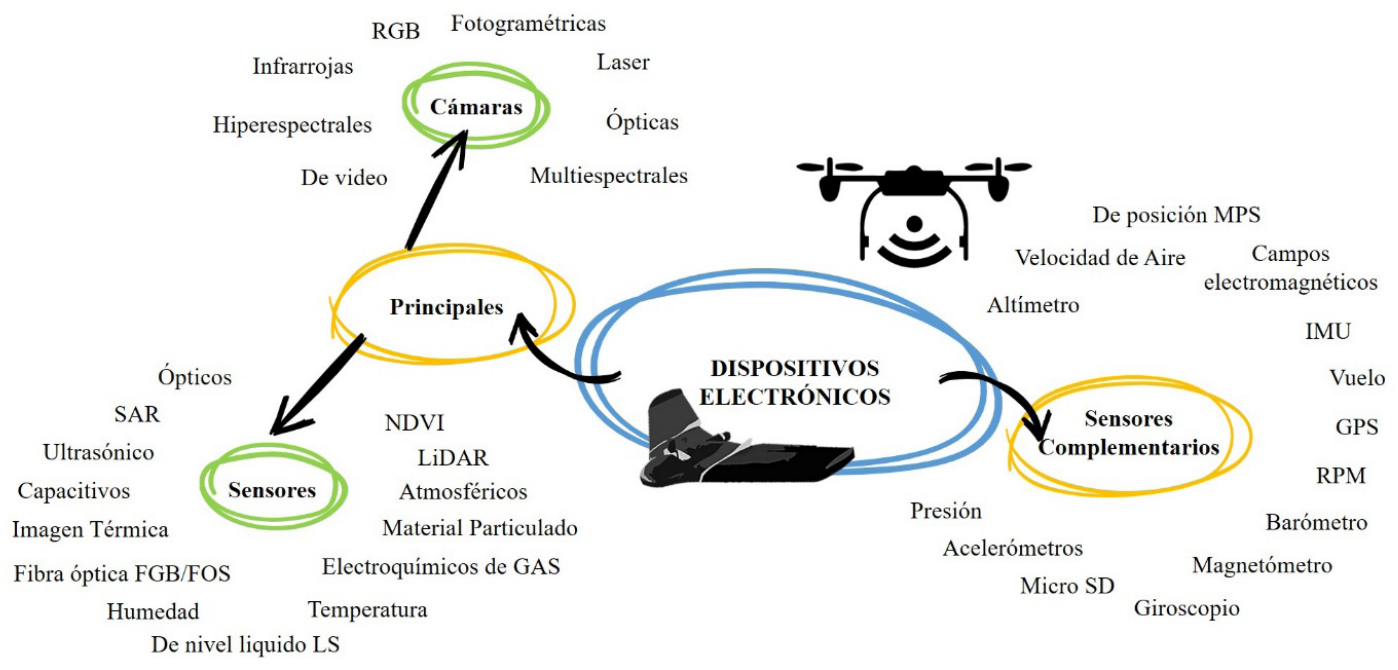

Fuente: Elaboración propia

Los dispositivos electrónicos se clasificaron en dos categorías, principales y sensores complementarios; donde los principales a su vez se subdividen en cámaras y sensores. Las cámaras comúnmente son utilizadas para la adquisición de imágenes de amplio espectro mediante herramientas ópticas e infrarrojas que permiten la creación de diagramas, mapas o esquemas de las zonas estudiadas y modelos de detección de formas, personas y animales [52,53,54]; los sensores tales como electroquímicos, atmosféricos y de material particulado, evalúan las variables presentes en investigaciones relacionadas con el medio ambiente y la agricultura; además sistemas láser, radares de apertura sintética (en inglés, Synthetic Aperture Radar SAR) y ultrasónicos a fin de determinar, durante el proceso de monitoreo, las zonas adecuadas donde se llevarán a cabo las mediciones $[55,56]$. Por otro lado, los sensores complementarios incorporados en estos vehículos mejoran el desplazamiento y posicionamiento durante los vuelos realizados con la finalidad de obtener la ubicación, aceleración, velocidad, altitud y orientación partiendo desde las unidades de medición inercial (en inglés, Inertial Measurement Unit IMU) sensores de revoluciones por minuto (RPM) y GPS [57, 58]. De esta manera, los componentes encontrados facilitan el desarrollo pleno de las misiones ejecutadas por los vehículos aéreos a escala en cada una de las aplicaciones encontradas.

Los sistemas de comunicación para la transmisión de información recolectada por el UAV se clasificaron en cuatro grupos, estableciendo de forma porcentual el uso de estos.

La primera clasificación presenta los sistemas basados en estaciones de control en tierra con un $58.43 \%$ del total de las revisiones realizadas de UAV que presentan comunicación y control directo con estas o con sistemas satelitales transmitiendo la información y las órdenes de manera bidireccional. La segunda son las comunicaciones inter - UAV se presenta un $5.62 \%$ que establecen conexiones inalámbricas fijas entre múltiples vehículos a fin de conocer aspectos como la posición, trayectoria y destino de los mismos permitiendo asignar una labor específica al dispositivo más cercano en zonas determinadas. La tercera son las comunicaciones móviles con un 20.22 \% que adaptan sistemas de comunicación a través de dispositivos móviles que permiten la recepción de la información obtenida por los UAV en tiempo real mediante softwares de monitoreo basados en Apps, desarrolladas en diferentes lenguajes de programación [59]. Así 
Vehículos aéreos no tripulados como alternativa de solución a los retos de innovación en diferentes campos de aplicación: una revisión de la literatura

mismo, estos vehículos incorporan sistemas de pilotaje autónomo para la realización de las rutas de vuelo preestablecidas y que pueden ser modificadas desde estas App. Sin embargo, la cuarta clasificación no presentaba la tecnología implementada en sus procesos de comunicación estableciendo un $15.73 \%$. Por último, los principales módulos de comunicación adaptados a las estructuras de los UAV son: WI-FI, XBEE, Zigbee, LoRaWAN, módulos de comunicaciones móviles (GSM, 3G y 4G) y Radiofrecuencia (en inglés, Radio Frecuency RF) [15, 60, 61, 62].

\section{Conclusiones}

Este trabajo realiza una revisión de la literatura basándose en la herramienta ToS y el método de revisión sistemática obteniendo una selección de 81 artículos de los cuales se destacó el uso de los UAV en aplicaciones como agricultura, ambiente, militar, mapeo y geociencias, destacándose la incorporación de sensores para mediciones remotas y de buena precisión del entorno circundante, demostrando que los UAV pueden ser una herramienta de bajo costo en comparación con las soluciones empleadas convencionalmente para estas aplicaciones, que se logra adaptar a las diferentes necesidades investigativas en múltiples sectores. Una de las principales ventajas de los UAV es la capacidad de obtener información rápida y de alta resolución con datos espaciales georreferenciados en entornos donde se hace difícil el acceso, sin embargo, la principal desventaja de estos dispositivos es la estabilidad, seguridad y la capacidad reducida de transporte de carga paga con bajos recursos por lo que dependiendo de la aplicación o uso se puede considerar emplear un tipo de UAV diferente.

En cuanto al diseño, el $58.82 \%$ emplean UAV multirrotor para entornos limitantes de espacio y despegues verticales, en casos que sea necesario recorrer zonas amplias en un corto periodo de tiempo el uso de los UAV de ala fija representa una mejor alternativa con $31.76 \%$, por otro lado, los UAV híbridos presentan un 1.18 \% de aplicabilidad en el ámbito militar. En consecuencia, el análisis de la documentación muestra que los vehículos aéreos tipo multirrotor son los más utilizados en cada área de aplicación, no obstante, el porcentaje restante no especificaba el tipo de vehículo implementado.

Adicionalmente, los UAV presentaron popularidad al usos de herramientas fotográficas con un $91.36 \%$ empleando cámaras para la adquisición de imágenes en diferentes espectros visuales a fin de validar el estado de un lugar o zona de estudio además de las facilidades de algunas características de estos vehículos como el peso, bajo costo, diseño y modelos estructurales que generan una versatilidad en la captura de imágenes ya sea en formatos RGB, multiespectrales, hiperespectrales o térmicas, demostrando que el uso de estos obtiene resultados eficientes, óptimos, rápidos, precisos y completos en comparación a otros dispositivos y métodos tradicionales.

Con las características encontradas se ha identificado que los UAV han aportado con un $25.93 \%$ en el sector ambiental, haciendo uso de estos dispositivos en estrategias forestales, estudios de agua y monitoreo del aire, seguidamente se encuentra el uso en la agricultura con un $22.22 \%$, aplicándolos principalmente para el desarrollo de mapas de rendimiento y fumigación de plagas, así mismo el campo de geociencias y mapeo presentan un $19.75 \%$ y $14.81 \%$ respectivamente, desarrollando diagramas en tercera dimensión para la evaluación de zonas rocosas y estudios cartográficos y topográficos. El uso de estos dispositivos para el área militar no tuvo el apogeo esperado demostrando una caída en las investigaciones con un $8.64 \%$, no obstante, el área de la logística muestra una nueva tendencia investigativa con un 8.64 \% desarrollando actividades de mensajería en el proceso de entrega y desplazamiento de paquetes, víveres y recursos médicos demostrándose con las pocas publicaciones revisadas en esta área que la logística de última milla 
y la humanitaria son temas potenciales de nuevo conocimiento y con retos a superar que van desde el uso de múltiples UAV, optimización de tiempos de desplazamiento, rutas aéreas y el desarrollo de estaciones de carga. Siendo estos aspectos retos a superar para ofrecer un mejor servicio al consumidor, reducir el daño ambiental y fortalecer el comercio electrónico llegando a lugares de difícil acceso mediante sistemas compactos y de menor consumo energético.

\section{Referencias Bibliográficas}

1. B. D. Song, K. Park, and J. Kim, "Persistent UAV delivery logistics: MILP formulation and efficient heuristic," Comput. Ind. Eng., vol. 120, no. February 2017, pp. 418-428, 2018, DOI: https://doi. org/10.1016/j.cie.2018.05.013.

2. L. D. M. Lam, A. Tang, and J. Grundy, "Heuristics-based indoor positioning systems: a systematic literature review," J. Locat. Based Serv., vol. 10, no. 3, pp. 178-211, 2016, DOI: https://doi.org/10.1080/ 17489725.2016.1232842.

3. S. Hayat, E. Yanmaz, and R. Muzaffar, "Survey on Unmanned Aerial Vehicle Networks for Civil Applications: A Communications Viewpoint," IEEE Commun. Surv. Tutorials, vol. 18, no. 4, pp. 26242661, 2016, DOI: https://doi.org/10.1109/COMST.2016.2560343.

4. D. Popescu, F. Stoican, L. Ichim, G. Stamatescu, and C. Dragana, "Collaborative UAV-WSN system for data acquisition and processing in agriculture," Proc. 2019 10th IEEE Int. Conf. Intell. Data Acquis. Adv. Comput. Syst. Technol. Appl. IDAACS 2019, vol. 1, pp. 519-524, 2019, DOI: https://doi.org/10.1109/ IDAACS.2019.8924424.

5. T. Niedzielski, "Applications of Unmanned Aerial Vehicles in Geosciences: Introduction," no. November, pp. 1-4, 2019, DOI: https://doi.org/10.1007/978-3-030-03171-8_1.

6. P. V. M. Maia, R. M. Santos, J. R. P. Vaz, M. O. Silva, and E. F. Lins, "Experimental study of three different airfoils applied to diffuser-augmented wind turbines," J. Urban Environ. Eng., vol. 12, no. 1, pp. 147-153, 2018, DOI: https://doi.org/10.4090/juee.

7. D. Lopez and Joana Andrea, "Marketing en Redes Sociales Online como herramienta de marketing Emprendedor," Univeridad Nacional de Colombia, 2016.

8. J. C. Marín, S. Robledo, and N. D. Duque, "Marketing emprendedor: una perspectiva cronologica utilizando tree of science," Civilizar Empres. y Econ., vol. 13, no. 1, pp. 113-123, 2017.

9. J. Toro and M. D. P. Rodríguez, "Formación en ética en las organizaciones: Revisión de la literatura," Inf. Tecnol., vol. 28, no. 2, pp. 167-180, 2017, DOI: https://doi.org/10.4067/S0718-07642017000200018.

10. C. Manterola, P. Astudillo, E. Arias, and N. Claros, "Revisiones sistemáticas de la literatura. Qué se debe saber acerca de ellas," Cir. Esp., vol. 91, no. 3, pp. 149-155, 2013, DOI: https://doi.org/10.1016/j. ciresp.2011.07.009.

11. E. L. García Alba, "Características y utilidad de las Revisiones Sistemáticas o Meta-análisis," Rev. Científica Cienc. Médica, vol. 16, no. 2, pp. 4-5, 2013.

12. J. Primicerio et al., "A flexible unmanned aerial vehicle for precision agriculture," Precis. Agric., vol. 13, no. 4, pp. 517-523, 2012, DOI: https://doi.org/10.1007/s11119-012-9257-6.

13. P. Hu, W. Guo, S. C. Chapman, Y. Guo, and B. Zheng, "Pixel size of aerial imagery constrains the applications of unmanned aerial vehicle in crop breeding," ISPRS J. Photogramm. Remote Sens., vol. 154, no. June 2018, pp. 1-9, 2019, DOI: https://doi.org/10.1016/j.isprsjprs.2019.05.008. 
14. N. Chebrolu, T. Labe, and C. Stachniss, "Robust long-term registration of UAV images of crop fields for precision agriculture," IEEE Robot. Autom. Lett., vol. 3, no. 4, pp. 3097-3104, 2018, DOI: https://doi. org/10.1109/LRA.2018.2849603.

15. S. Bhandari, A. Raheja, R. L. Green, and D. Do, "Towards collaboration between unmanned aerial and ground vehicles for precision agriculture," Auton. Air Gr. Sens. Syst. Agric. Optim. Phenotyping II, vol. 10218, p. 1021806, 2017, DOI: https://doi.org/10.1117/12.2262049.

16. X. Q. Zhang et al., "Application of Multi-rotor Unmanned Aerial Vehicle Application in Management of Stem Borer (Lepidoptera) in Sugarcane," Sugar Tech, vol. 21, no. 5, pp. 847-852, 2019, DOI: https://doi. org/10.1007/s12355-018-0695-y.

17. P. Venkata Subba Rao and S. R. Gorantla, "Design and Modelling of anAffordable UAV Based Pesticide Sprayer in Agriculture Applications," 5th Int. Conf. Electr. Energy Syst. ICEES 2019, vol. 360, no. February, pp. 1-4, 2019, DOI: https://doi.org/10.1109/ICEES.2019.8719237.

18. K. Kuru, D. Ansell, W. Khan, and H. Yetgin, "Analysis and Optimization of Unmanned Aerial Vehicle Swarms in Logistics: An Intelligent Delivery Platform," IEEE Access, vol. 7, no. c, pp. 15804-15831, 2019, DOI: https://doi.org/10.1109/ACCESS.2019.2892716.

19. J. Lee et al., "A mission management system for complex aerial logistics by multiple unmanned aerial vehicles in MBZIRC 2017," J. F. Robot., vol. 36, no. 5, pp. 919-939, 2019, DOI: https://doi.org/10.1002/ rob. 21860 .

20. H. Ni, X. Deng, B. Gong, and P. Wang, “Design of regional logistics system based on unmanned aerial vehicle," Proc. 2018 IEEE 7th Data Driven Control Learn. Syst. Conf. DDCLS 2018, pp. 1045-1051, 2018, DOI: https://doi.org/10.1109/DDCLS.2018.8515965.

21. C. Sutheerakul, N. Kronprasert, M. Kaewmoracharoen, and P. Pichayapan, "Application of Unmanned Aerial Vehicles to Pedestrian Traffic Monitoring and Management for Shopping Streets," Transp. Res. Procedia, vol. 25, pp. 1717-1734, 2017, DOI: https://doi.org/10.1016/j.trpro.2017.05.131.

22. M. Golabi, S. M. Shavarani, and G. Izbirak, "An edge-based stochastic facility location problem in UAVsupported humanitarian relief logistics: a case study of Tehran earthquake," Nat. Hazards, vol. 87, no. 3, pp. 1545-1565, 2017, DOI: https://doi.org/10.1007/s11069-017-2832-4.

23. A. Vehicles, "Current and future UAV military users and applications," Air Sp. Eur., vol. 1, no. 5-6, pp. 51-58, 1999, DOI: https://doi.org/10.1016/s1290-0958(00)88871-1.

24. M. A. Ma'Sum et al., "Simulation of intelligent Unmanned Aerial Vehicle (UAV) for military surveillance," 2013 Int. Conf. Adv. Comput. Sci. Inf. Syst. ICACSIS 2013, pp. 161-166, 2013, DOI: https://doi.org/10.1109/ ICACSIS.2013.6761569.

25. M. Zhang, H. Li, G. Xia, W. Zhao, S. Ren, and C. Wang, "Research on the Application of Deep Learning Target Detection of Engineering Vehicles in the Patrol and Inspection for Military Optical Cable Lines by UAV," Proc. - 2018 11th Int. Symp. Comput. Intell. Des. Isc. 2018, vol. 1, pp. 97-101, 2018, DOI: https:// doi.org/10.1109/ISCID.2018.00029.

26. S. J. Levulis, P. R. DeLucia, and S. Y. Kim, “Effects of Touch, Voice, and Multimodal Input, and Task Load on Multiple-UAV Monitoring Performance During Simulated Manned-Unmanned Teaming in a Military Helicopter," Hum. Factors, vol. 60, no. 8, pp. 1117-1129, 2018, DOI: https://doi. org/10.1177/0018720818788995.

27. D. Orfanus, E. P. De Freitas, and F. Eliassen, "Self-Organization as a Supporting Paradigm for Military UAV Relay Networks," IEEE Commun. Lett., vol. 20, no. 4, pp. 804-807, 2016, DOI: https://doi.org/10.1109/ LCOMM.2016.2524405. 
28. J. E. Márquez Díaz, "Seguridad metropolitana mediante el uso coordinado de Drones," Ing. USBMed, vol. 9, no. 1, p. 39, 2018, DOI: https://doi.org/10.21500/20275846.3299.

29. R. Kuntz Rangel, J. L. Freitas, and V. Antonio Rodrigues, "Development of a Multipurpose Hydro Environmental Tool using Swarms, UAV and USV," IEEE Aerosp. Conf. Proc., vol. 2019-March, 2019, DOI: https://doi.org/10.1109/AERO.2019.8741624.

30. M. A. Boon, A. P. Drijfhout, and S. Tesfamichael, "Comparison of a fixed-wing and multi-rotor UAV for environmental mapping applications: A case study," Int. Arch. Photogramm. Remote Sens. Spat. Inf. Sci. - ISPRS Arch., vol. 42, no. 2W6, pp. 47-54, 2017, DOI: https://doi.org/10.5194/isprs-archives-XLII2-W6-47-2017.

31. Y. Lu, D. Macias, Z. S. Dean, N. R. Kreger, and P. K. Wong, "A UAV-Mounted Whole Cell Biosensor System for Environmental Monitoring Applications," IEEE Trans. Nanobioscience, vol. 14, no. 8, pp. 811-817, 2015, DOI: https://doi.org/10.1109/TNB.2015.2478481.

32. K. F. Flynn and S. C. Chapra, "Remote sensing of submerged aquatic vegetation in a shallow nonturbid river using an unmanned aerial vehicle," Remote Sens., vol. 6, no. 12, pp. 12815-12836, 2014, DOI: https://doi.org/10.3390/rs61212815.

33. J. Witczuk, S. Pagacz, A. Zmarz, and M. Cypel, "Exploring the feasibility of unmanned aerial vehicles and thermal imaging for ungulate surveys in forests - preliminary results," Int. J. Remote Sens., vol. 39, no. 15-16, pp. 5504-5521, 2018, DOI: https://doi.org/10.1080/01431161.2017.1390621.

34. R. Woellner and T. C. Wagner, "Saving species, time and money: Application of unmanned aerial vehicles (UAVs) for monitoring of an endangered alpine river specialist in a small nature reserve," Biol. Conserv., vol.233, no. October 2018, pp.162-175, 2019, DOI: https://doi.org/10.1016/j.biocon.2019.02.037.

35. L. Mead and M. Arthur, "Environmental condition in British moorlands: quantifying the life cycle of Calluna vulgaris using UAV aerial imagery," Int. J. Remote Sens., vol. 41, no. 2, pp. 573-583, 2020, DOI: https://doi.org/10.1080/2150704X.2019.1646931.

36. J. N. Hird et al., "Use of unmanned aerial vehicles for monitoring recovery of forest vegetation on petroleum well sites," Remote Sens., vol. 9, no. 5, 2017, DOI: https://doi.org/10.3390/rs9050413.

37. S. Jayathunga, T. Owari, and S. Tsuyuki, "The use of fixed-wing UAV photogrammetry with LiDAR DTM to estimate merchantable volume and carbon stock in living biomass over a mixed coniferbroadleaf forest," Int. J. Appl. Earth Obs. Geoinf., vol. 73, no. August, pp. 767-777, 2018, DOI: https://doi. org/10.1016/j.jag.2018.08.017.

38. V. Sherstjuk and M. Zharikova, "Fire-front recognition in UAV-based forest-fire monitoring system using fuzzy rough soft sets," 2019 IEEE 2nd Ukr. Conf. Electr. Comput. Eng. UKRCON 2019 - Proc., pp. 1091-1096, 2019, DOI: https://doi.org/10.1109/UKRCON.2019.8879829.

39. M. N. Saadat and M. N. Husen, "An application framework for forest fire and haze detection with data acquisition using unmanned aerial vehicle," ACM Int. Conf. Proceeding Ser., 2018, DOI: https://doi. org/10.1145/3164541.3164624.

40. J. Fernández-Hernandez, D. González-Aguilera, P. Rodríguez-Gonzálvez, and J. Mancera-Taboada, "Image-Based Modelling from Unmanned Aerial Vehicle (UAV) Photogrammetry: An Effective, LowCost Tool for Archaeological Applications," Archaeometry, vol. 57, no. 1, pp. 128-145, 2015, DOI: https:// doi.org/10.1111/arcm.12078.

41. A. Y. M. Lin, A. Novo, S. Har-Noy, N. D. Ricklin, and K. Stamatiou, "Combining GeoEye-1 Satellite Remote Sensing, UAV Aerial Imaging, and Geophysical Surveys in Anomaly Detection Applied to Archaeology," IEEE J. Sel. Top. Appl. Earth Obs. Remote Sens., vol. 4, no. 4, pp. 870-876, 2011, DOI: https://doi. org/10.1109/JSTARS.2011.2143696. 
42. A. A. Doshi, A. J. Postula, A. Fletcher, and S. P. N. Singh, "Development of micro-UAV with integrated motion planning for open-cut mining surveillance," Microprocess. Microsyst., vol. 39, no. 8, pp. 829835, 2015, DOI: https://doi.org/10.1016/j.micpro.2015.07.008.

43. L. Ge, X. Li, and A. H. M. Ng, "UAV for mining applications: A case study at an open-cut mine and a longwall mine in New South Wales, Australia," Int. Geosci. Remote Sens. Symp., vol. 2016-Novem, no. 1, pp. 5422-5425, 2016, DOI: https://doi.org/10.1109/IGARSS.2016.7730412.

44. Y. Fang, Z. Hu, L. Xu, A. Wong, and D. A. Clausi, "Estimation Of Iron Concentration In Soil Of A Mining Area From Uav-Based Hyperspectral Imagery," in 2019 10th Workshop on Hyperspectral Imaging and Signal Processing: Evolution in Remote Sensing (WHISPERS), 2019, pp. 1-5, DOI: https://doi.org/10.1109/ WHISPERS.2019.8920973.

45. A. Ranjan, B. Panigrahi, H. B.Sahu, and P. Misra, “SkyHelp:Leveraging UAVsfor emergency communication support in deep open pit mines," 2018 10th Int. Conf. Commun. Syst. Networks, COMSNETS 2018, vol. 2018-Janua, no. 1, pp. 546-548, 2018, DOI: https://doi.org/10.1109/COMSNETS.2018.8328269.

46. O. G. Ajayi, M. Palmer, and A. A. Salubi, "Modelling farmland topography for suitable site selection of dam construction using unmanned aerial vehicle (UAV) photogrammetry," Remote Sens. Appl. Soc. Environ., vol. 11, no. July, pp. 220-230, 2018, DOI: https://doi.org/10.1016/j.rsase.2018.07.007.

47. K. N. Tahar, A. Ahmad, W. A. A. Wan Mohd Akib, and W. M. N. Wan Mohd, "Aerial mapping using autonomous fixed-wing unmanned aerial vehicle," Proc. - 2012 IEEE 8th Int. Colloq. Signal Process. Its Appl. CSPA 2012, pp. 164-168, 2012, DOI: https://doi.org/10.1109/CSPA.2012.6194711.

48. T. C. Su, "Multispectral sensors carried on unmanned aerial vehicle (UAV) for trophic state mapping of the small reservoir in Kinmen, Taiwan," Int. Geosci. Remote Sens. Symp., vol. 2015-Novem, pp. 53485351, 2015, DOI: https://doi.org/10.1109/IGARSS.2015.7327043.

49. O. Wigmore, B. Mark, J. McKenzie, M. Baraer, and L. Lautz, "Sub-metre mapping of surface soil moisture in proglacial valleys of the tropical Andes using a multispectral unmanned aerial vehicle," Remote Sens. Environ., vol. 222, no. January 2018, pp. 104-118, 2019, DOI: https://doi.org/10.1016/j. rse.2018.12.024.

50. F. H. Yeh, C. J. Huang, J. Y. Han, and L. Ge, "Modeling Slope Topography Using Unmanned Aerial Vehicle Image Technique," MATEC Web Conf., vol. 147, pp. 1-6, 2018, DOI: https://doi.org/10.1051/ matecconf/201814707002.

51. M. Yu, Y. Huang, J. Zhou, and L. Mao, "Modeling of landslide topography based on micro-unmanned aerial vehicle photography and structure-from-motion," Environ. Earth Sci., vol. 76, no. 15, 2017, DOI: https://doi.org/10.1007/s12665-017-6860-x.

52. S. J. Hong, Y. Han, S. Y. Kim, A. Y. Lee, and G. Kim, "Application of deep-learning methods to bird detection using unmanned aerial vehicle imagery," Sensors (Switzerland), vol. 19, no. 7, pp. 1-16, 2019, DOI: https://doi.org/10.3390/s19071651.

53. P. J. Zarco-Tejada, L. Suárez, J. A. J. Berni, E. Fereres, J. A. J. Berni, and P. J. Zarco-Tejada, "Thermal and Narrowband Multispectral Remote Sensing for Vegetation Monitoring From an Unmanned Aerial Vehicle Improved Evapotranspiration using Unmanned Aerial Vehicles View project High throughput and remote trait measurement View project Thermal and Nar," leee Trans. Geosci. Remote Sens., vol. 47, no. 3, pp. 722-738, 2009, DOI: https://doi.org/10.1109/TGRS.2008.2010457.

54. V. Trukhachev, S. Oliinyk, T. Lesnyak, and N. Zlyidnev, "Application of unmanned aerial vehicles for remote estimation of pasture fertility while growing Dzhalginsky merino sheep," Eng. Rural Dev., vol. 18, no. 1, pp. 1673-1679, 2019, DOI: https://doi.org/10.22616/ERDev2019.18.N465. 
55. S. S. Esfahlani, "Mixed reality and remote sensing application of unmanned aerial vehicle in fire and smoke detection," J. Ind. Inf. Integr., vol. 15, no. November 2018, pp. 42-49, 2019, DOI: https://doi. org/10.1016/j.jii.2019.04.006.

56. C. Liu, X. Liu, X. Peng, E. Wang, and S. Wang, "Application of 3D-DDA integrated with unmanned aerial vehicle-laser scanner (UAV-LS) photogrammetry for stability analysis of a blocky rock mass slope," Landslides, vol. 16, no. 9, pp. 1645-1661, 2019, DOI: https://doi.org/10.1007/s10346-019-01196-6.

57. S. Siebert and J. Teizer, “Mobile 3D mapping for surveying earthwork projects using an Unmanned Aerial Vehicle (UAV) system," Autom. Constr., vol. 41, pp. 1-14, 2014, DOI: https://doi.org/10.1016/j. autcon.2014.01.004.

58. T. C. Su and H. T. Chou, "Application of multispectral sensors carried on unmanned aerial vehicle (UAV) to trophic state mapping of small reservoirs: A case study of Tain-Pu reservoir in Kinmen, Taiwan," Remote Sens., vol. 7, no. 8, pp. 10078-10097, 2015, DOI: https://doi.org/10.3390/rs70810078.

59. D. G. Schmale III, B. R. Dingus, and C. Reinholtz, "Development and application of an autonomous unmanned aerial vehicle for precise aerobiological sampling above agricultural fields," J. F. Robot., vol. 25, no. 3, pp. 133-147, Mar. 2008, DOI: https://doi.org/10.1002/rob.20232.

60. R. Avellaneda, S. Cabrera, P. A. Martínez, y C. G. Donoso Albarracín, "Apoyo tecnológico para la fidelización y captación de nuevos clientes por medio de una aplicación móvil", Investigación e Innovación en Ingenierías, vol. 5, n.o 1, pp. 92-101, 2017. DOI: https://doi.org/10.17081/invinno.5.1.2618

61. D. Henao - león, A. Camilo Báez - Alarcón, y J. Bethsaid Pedroza - Rojas, “Metodología para determinar la viabilidad de generación de energía eléctrica por medio del recurso eólico", Revista Investigación e Innovación en Ingenierías, vol. 6, nº. 2, 2018. DOI: https://doi.org/10.17081/invinno.6.2.3108

62. M. Piras ,G. Taddia ,M. G. Forno ,M. Gattiglio ,I. Aicardi ,P. Dabove, et al., “Detailed geological mapping in mountain areas using an unmanned aerial vehicle: application to the Rodoretto Valley, NW Italian Alps," Geomatics, Nat. Hazards Risk, vol. 8, no. 1, pp. 137-149, 2017, DOI: https://doi.org/10.1080/1947 5705.2016 .1225228 . 